\title{
Abstracts from the ASENT 19th Annual Meeting
}

Published online: 19 June 2017

(C) The American Society for Experimental NeuroTherapeutics, Inc. 2017

Title: Extrapolating Anti-Epileptic Drug Efficacy Data from Adults to Children; Results from the PEACE Initiative

Authors: O'Neill D'Cruz MD, MBA; John Pellock - OD Consulting and Neurological Services, PLLC

\begin{abstract}
:
Objective: Initial approval of anti-epileptic drugs (AEDs) for seizure types common in adults and children is based on trials conducted in adults; off-label use limits enrollment in pediatric clinical trials. Pediatric Epilepsy Academic Consortium for Extrapolation (PEACE) investigated the scientific basis for extrapolation of efficacy of AEDs from adults to children with the goal of expediting AED approvals for pediatric use.
\end{abstract}

Methods: The PEACE initiative was a collaborative effort between academic, regulatory and industry partners to formulate the biological basis of extrapolation, compare clinical trial findings and assess placebo effects and concentrationresponse relationships for various AEDs. Trial data for various seizure types, age range for extrapolation, and regulatory guidelines were reviewed to develop scientific consensus and propose revision of regulatory policies.

Results: Biological basis for extrapolation of efficacy from adults to children 4 years and older for focal seizures was supported by preclinical, neurophysiological and clinical data. Clinical trial data showed comparable treatment effect sizes between adults and children for adjunctive therapy of focal seizures for five drugs (gabapentin, oxcarbazepine, lamotrigine, topiramate and levetiracetam); placebo responses and concentrationresponse relationships between adults and children for individual drugs were comparable. Findings were reviewed by the ILAE Regulatory Affairs Task Force
(RATF) and European, US and Japanese regulatory agencies.

Conclusions: PEACE proposal for extrapolation of efficacy from adults to children 4 years and older for adjunctive use of AEDs for treatment of focal seizures was endorsed by the ILAE RATF. Regulatory agencies revised and updated policies to accept extrapolation as proposed by the PEACE initiative.

$* * *$

Title: A pH-sensitive Doxorubicin Prodrug Based on Lactoferrin-conjugated Hyaluronic Acid for Glioma-targeted Drug Delivery

Authors: Ying Peng PhD; Yatao Yin - Department of Neurology, Sun Yat-Sen Memorial Hospital; Sun Yat-sen University

\begin{abstract}
:
Gliomas are the most common and lethal type of primary malignant brain tumor, with current treatments having limited impact on disease progression. The effective treatment is hindered by the poor transport across the blood brain barrier (BBB) and the low penetration across the blood-tumor barrier (BTB). In this sthdy, a novel macromolecular prodrug strategy was developed. Doxorubicin (DOX), a commonly used anticancer drug was firstly conjugated onto an endogenous glycosaminoglycan hyaluronic acid (HA) by an acid-labile hydrazone linkage, and was functionalized with lactoferrin (Lf), a specific ligand of low-density lipoprotein receptor-related protein (LRP), which could mediate the system to cross the BBB and target to glioma cells. In vitro, the release of DOX from HA-DOX was $\mathrm{pH}$ dependent. At lower $\mathrm{pH}$ 5.0, the release of DOX was
\end{abstract}


much quicker than that at $\mathrm{pH} 6.0$ and $\mathrm{pH}$ 7.4. Compared with free durg DOX, enhanced cellular uptake in both U87 and C6 cells, increased cytotoxicity of the loaded DOX was achieved by the dual-targeting LfHA-DOX in vitro. In vivo, enhanced accumulation of Lf-HA-DOX in the glioma was observed by real time fluorescence image. Correspondingly, glioma-bearing mice treated with Lf-HA-DOX displayed the longest median survival time, which was 2-fold longer than that of saline group. In conclusion, Lf-HA-DOX was able to significantly increase drug delivery to the glioma, which might provide a promising strategy for antiglioma therapy.

\section{$* * *$}

Title: Oral Administration of Erythromycin Decreases RNA Toxicity in Myotonic Dystrophy

Authors: Hideki Mochizuki MD,PhD; Masayuki Nakamori; Krzysztof Sobczak - Osaka University

\begin{abstract}
:
Objective: Myotonic dystrophy type 1 (DM1) is caused by the expansion of a CTG repeat in the 32 untranslated region of DMPK. The transcripts containing an expanded CUG repeat (CUGexp) result in a toxic gain-offunction by forming ribonuclear foci that sequester the alternative splicing factor MBNL1. Although several small molecules reportedly ameliorate RNA toxicity, none are ready for clinical use because of the lack of safety data. Here we undertook a drug-repositioning screen to identify a safe and effective small molecule for upcoming clinical trials of DM1.
\end{abstract}

Methods: We examined the potency of small molecules in inhibiting the interaction between CUGexp and MBNL1 by in vitro sequestration and fluorescent titration assays. We studied the effect of lead compounds in DM1 model cells by evaluating foci reduction and splicing rescue. We also tested their effects on missplicing and myotonia in DM1 model mice.

Results: Of the 20 FDA-approved small molecules tested, erythromycin showed the highest affinity to CUGexp and a capacity to inhibit its binding to MBNL1. Erythromycin decreased foci formation and rescued missplicing in DM1 cell models. Both systemic and oral administration of erythromycin in the DM1 model mice showed splicing reversal and improvement of myotonia with no toxicity. Long-term oral administration of erythromycin at the dose used in humans also improved the splicing abnormality in the DM1 model mice. Interpretation: Oral erythromycin treatment, which has been widely used in human with excellent tolerability, may be a promising therapy for DM1.
Financial Support: This work was supported by Grant-inAid for Young Scientists (Start-up) and Grant-in-Aid for Challenging Exploratory Research from Japan Society for the Promotion of Science.

$* * *$

Title: Inhibiting the Proliferation of Patient-Derived Glioblastoma Multiforme (GBM) Using Leaf Extract of Bacopa monnieri

Authors: Tejas Athni, Student (11th Grade) - Stratford Academy (High School); Brajesh Vaidya; Nirmal Joshee, $\mathrm{PhD}$ - Fort Valley State University; Prahlad Parajuli, PhD Wayne State University

\section{Abstract:}

Glioblastoma multiforme (GBM) is the most common and aggressive type of primary brain tumor. Patients with GBM have a median survival time of 15 months. Treatments currently available for GBM are quite limited and research into developing novel therapeutics is warranted. Currently, the thrust of GBM research is in the fields of immunotherapy, chemotherapy, and vaccination therapy.

Bacopa monnieri, a species of herbaceous perennials, has been shown to exhibit numerous positive effects on the CNS. Phytochemical analysis of Bacopa has revealed high concentrations of bioactive constituents known as terpenoids, which have anti-tumorigenic properties. Considering these properties of terpenoids, it was hypothesized that Bacopa may inhibit the proliferation of GBM cells in vitro. The objective was to prepare leaf extract of Bacopa monnieri and assess its effect on GBM cells.

Methanolic extract of Bacopa leaves was applied to patient-derived GBM cells and U-87 MG cell line in vitro. Tested extract concentrations ranged from 4 to $300 \mu \mathrm{g} / \mathrm{mL}$. After $72 \mathrm{hrs}$ of exposure, GBM cell proliferation was analyzed through a colorimetric WST-1 tetrazolium reduction assay.

The results revealed that cell proliferation was inhibited by the Bacopa extract in both patient-derived GBM cells and the U-87 MG cell line. The IC50 (dose for $50 \%$ inhibition of proliferation) range was found to be between 11 and $33 \mu \mathrm{g} / \mathrm{mL}$ for patient-derived GBM and between 33 and $100 \mu \mathrm{g} / \mathrm{mL}$ for U-87 MG. Data analysis revealed statistically-significant F-values of 282.809 and 28.911 for the patient-derived GBM and the U-87 MG cells, respectively. The $P$-value was less than 0.001 for both.

In conclusion, Bacopa leaf extract does indeed inhibit the proliferation of patient-derived GBM cells as well as the U-87 MG cell line, a finding which has never been previously reported. This study presents a potential novel approach to the treatment of GBM. If the 
bioactive compound responsible for the observed inhibitive phenomenon can be identified from the leaf extract and safely tested in animal trials and human clinical trials, Bacopa monnieri has the potential to be used as a novel adjuvant treatment modality for GBM, hopefully improving survival rate of afflicted patients.

\section{$* * *$}

Title: TAK-063, A Novel Phosphodiesterase 10A Inhibitor, Protects Striatal Neurons from Degeneration and Ameliorates Behavioral Deficits in the R6/2 Mouse Model of Huntington's Disease

Authors: Akina Harada; Kazunori Suzuki; Haruhide Kimura - Takeda Pharmaceutical Company Limited

\begin{abstract}
:
Huntington's disease (HD) is an inherited neurodegenerative disorder associated with motor dysfunctions and cognitive impairments. HD is characterized by progressive loss of striatal medium spiny neurons (MSNs), which constitute the direct and indirect pathways. Impairment of cAMP/cGMP signaling is hypothesized as the molecular mechanism underlying degeneration of MSNs. Phosphodiesterase 10A (PDE10A) is selectively expressed in MSNs and degrades both cAMP and cGMP; thus, PDE10A inhibition can restore impaired cAMP/cGMP signaling. Compared with other PDE10A inhibitors, TAK-063, a novel PDE10A inhibitor, showed comparable activation of the indirect pathway MSNs, while it produced partial activation of the direct pathway MSNs by its faster off-rate property. Given that indirect pathway MSNs are more vulnerable to degeneration than direct pathway MSNs in HD, TAK-063 with the indirect pathway MSN-biased activation pattern may be favorable without unbalanced activation of MSNs as a therapeutic agent for HD.

In this study, we report the effects of TAK-063 on striatal neurodegeneration and behavioral deficits in the R6/2 mouse model of HD. TAK- 063 at 0.5 or $5 \mathrm{mg} / \mathrm{kg} /$ day was orally administered to R6/2 mice from $4.5,5$ to 12 weeks of age, and the effects of TAK-063 on phenotypes of R6/2 mice were characterized over this period. TAK-063 at 0.5 and $5 \mathrm{mg} / \mathrm{kg} / \mathrm{day}$ significantly and dose-dependently suppressed the reduction of striatal BDNF protein levels. TAK-063 significantly prevented striatal neurodegeneration and suppressed an increase in seizure frequency at $5 \mathrm{mg} / \mathrm{kg} / \mathrm{day}$, but it did not prevent the suppression of body weight gain. As for motor deficits, TAK-063 dose-dependently suppressed the development of clasping behavior and significantly improved dereased motor activity in the open field at 5 $\mathrm{mg} / \mathrm{kg} /$ day, but it did not improve the impairment in motor coordination on the rotarod. Regarding cognitive function,
\end{abstract}

TAK-063 significantly improved deficits in procedural learning in the water T-maze at $5 \mathrm{mg} / \mathrm{kg} / \mathrm{day}$, but was ineffective for deficits in contextual memory in contextual fear conditioning. These results suggest that repeated treatment with TAK-063 reduces striatal neurodegeneration and ameliorates behavioral deficits in R6/2 mice.

Financial Support: This work was supported by Takeda Pharmaceutical Company Limited. All authors are employees of Takeda Pharmaceutical Company Limited.

***

Title: Adverse Event Reporting in Clinical Trials: Time for Change

Authors: Christopher Kenney MD; Stephen Bandak - Acorda Therapeutics; Karl D Kieburtz, University of Rochester School of Medicine and Clintrex LLC; C Warren Olanow, Clintrex LLC and Mount Sinai School of Medicine

\begin{abstract}
:
Background: Adverse event (AE) reporting in clinical trials typically describes the frequency of specific events; hence, transient events may be counted as much as persistent events. This contrasts with the detailed reporting of efficacy measures, making risk/benefit assessment potentially inaccurate. To assess the clinical implication of this issue, we examined the frequency and temporal pattern of dyskinesia, an expected $\mathrm{AE}$, in a tozadenant clinical study to determine whether a closer understanding of the temporal profile of this $\mathrm{AE}$ would better inform the risk/benefit assessment of tozadenant.
\end{abstract}

Methods: Tozadenant is an adenosine A2A antagonist under investigation as an adjunct to levodopa in patients with Parkinson's disease experiencing motor fluctuations. A 12 -week phase $2 \mathrm{~b}$ study of tozadenant $60 \mathrm{mg}$, $120 \mathrm{mg}, 180 \mathrm{mg}, 240 \mathrm{mg}$ BID vs placebo was conducted in levodopa-treated patients experiencing e $2.5 \mathrm{~h} /$ day OFF time (Hauser RA, et al, Lancet Neurol 2014;13:767-776.) Dyskinesias were recorded as AEs at each visit, and included assessments of severity, relationship to study drug, and the dates of occurrence.

Results: 420 patients were randomized and received one or more doses of study drug. The frequency of dyskinesia-AE was $8.3 \%$ for placebo, vs $14.1 \%, 15.9 \%, 20.0 \%$, and $20.2 \%$ for tozadenant $60,120,180$, and $240 \mathrm{mg}$, respectively. The low-dose tozadenant groups (60 and $120 \mathrm{mg}$ ) had a dyskinesia-AE prevalence higher than placebo only during the first 3 weeks of treatment, and comparable to placebo thereafter. In the high-dose groups $(180$ and $240 \mathrm{mg})$, the dyskinesia-AE prevalence was consistently higher than placebo throughout the treatment duration. Improvement in OFF 
time was noted for all treatment groups and persisted throughout the study.

Interpretation: The temporal profile of dyskinesia as an $\mathrm{AE}$ was more informative than overall frequency. By standard $\mathrm{AE}$ reporting, clinical improvement was associated with an increase in dyskinesia at all dosages, compared with placebo. Analysis of the pattern noted that reports of dyskinesia were transient with the lower dose groups, even though motor benefits persisted. We propose that routine reporting of AEs should include information regarding frequency and duration or pattern, so as to better inform on the balance between risk and benefit.

Financial Support: Acorda Therapeutics.

\section{$* * *$}

Title: HTT-AS Suppression of HTT: A Potential Therapeutic Approach for Huntington's Disease

Authors: Pan P. Li, PhD; Ying Song; Xin Sun; Wang Zheng; Wenjuan Ye, BS; Xin Hu, PhD; Marc Ferrer, PhD; Dobrila D. Rudnicki, PhD; Russell L. Margolis, MD - Division of Neurobiology, Department of Psychiatry, Johns Hopkins University School of Medicine, Baltimore, MD

\footnotetext{
Abstract:

Huntington's disease (HD) is an autosomal dominant disorder caused by a CAG repeat expansion in the first exon of the Huntingtin (HTT) gene. We have previously found that Huntingtin antisense (HTT-AS), an endogenous gene on the opposite strand of HTT, is widely expressed at low levels in the brain and, when overexpressed, suppresses HTT expression. Here we test the hypothesis that HTT-AS itself, and the components that enhance its expression, will provide novel therapeutic targets for suppression of HTT expression and hence treatment of HD.

To investigate, this hypothesis, we generated HEK293 cell lines in which the HTT-AS promoter linked to both firefly and Renilla luciferase coding regions was integrated into the genome. After careful screening, cell line HTTAS- 8 was selected for use in a pilot high throughput screen (HTS). After optimization, a pilot HTS using the LOPAC 1280 compound library (a set of functionally characterized small molecules) was performed. This assay identified 17 small molecule agents that increased HTT-AS promoter activity. Efforts are underway to validate the effect of these small molecules on reducing HTT expression in HD lymphoblastoid cells.

The success of this pilot screen is consistent with our hypothesis that small molecules can be used to decrease HTT expression by increasing HTT-AS expression. To pursue this investigation further, we are now 1) expanding our HTS to larger compound libraries, 2) developing cell models to determine the quantitative relationship between HTT-AS expression and HTT suppression, and 3) exploring a pilot HTS to detect small molecules that directly suppress HTT promoter activity.
}

Financial Support: Hereditary Disease Foundation, CHDI, $\mathrm{NIH}$, and ABCD Charitable Trust

$* * *$

Title: Strategy to Improve Clinical Development

Author: Harumasa Nakamura, MD; Shin'ichi Takeda, National Institute of Neuroscience, National Center of Neurology and Psychiatry

\section{Abstract:}

Real-world evidence is derived from Real-world data (RWD), and can inform drug/device development, research on outcomes and health care systems, safety surveillance, well-controlled effectiveness studies, and patient care, in the nervous system. Clinical Innovation Network (CIN) is new a concept supported by Ministry of Health, Labour and Welfare Japan to improve the environment of the clinical development that enables efficient clinical trial by utilizing patient registry that is a primary key source of RWD. Usefulness of patient registry in clinical trial/ research in the clinical development can be broadly outlined as follows; market research, survey on the feasibility of clinical trial, creation of protocol, recruiting patients to clinical trial, establishing control group in non randomized control study, postmarketing survey and safety measures. In doing this, it is required to establish a network of the related institutions and to form the clinical study consortium in cooperation with the industry and academia, as well as regulatory agency. The following issues were considered as common problems regardless of the individual disease area. 1) Gathering information about existing patient registries, analysis and listing in the form that is easy to use for pharmaceutical companies and others, 2) Ethical issues and how to handle personal information, 3) Standardization of registry items and information technology system, 4) How to use and apply patients' registry data to clinical trial/clinical research. In addition, infrastructure of the central support in order to promote CIN and the function of "One-Stop" is demanded by pharmaceutical companies. Furthermore, new registries are required based on the regulatory complaint for natural history as control groups in clinical trial and the source of post-marketing survey to provide efficacy and safety measures as RWD.

***

Title: Nanodendrimer Therapy for Adrenoleukodystrophy and Other Genetic Disorders of Myelination

Authors: Ali Fatemi MD, MBA; Christina Mertz; Bela Turk - Kennedy Krieger Institute, Johns Hopkins University; Kannan Rangaramanujam; Sujatha Kannan - Center for Nanomedicine, Ophthalmology, Johns Hopkins University

\section{Abstract:}

X-linked adrenoleukodystrophy (ALD) is a demyelinating disorder with an incidence of 1:17,000 males, caused by 
a mutation of the $\mathrm{ABCD}$ gene, resulting in high levels of circulating very long chain fatty acids (VLCFAs). Around $35 \%$ of boys with X-ALD develop the childhood cerebral form (ccALD) that is characterized by oxidative stress, neuroinflammation and microglial activation leading to a rapidly progressive cerebral demyelination that is almost fatal within 2-3 years. The only therapy that is currently available is hematopoietic stem cell transplant (HSCT) that is effective in arresting neuroinflammation and demyelination but only when provided very early in the course of the disease. Therefore, adjuvant treatments are necessary for patients who are diagnosed late or are not eligible for HSCT, and for arresting disease progression during HSCT. We have previously shown that dendrimer $\mathrm{N}$ acetyl cysteine nanoparticles (D-NAC) target activated microglia/ macrophages in the brain decreasing inflammation and oxidative stress leading to improved motor function in pre-clinical models of neuroinflammation.

Objective: We hypothesize that macrophages derived from monocytes of patients with ALD are activated to a pro- inflammatory state when stimulated with VLCFAs and that treatment with D-NAC ex vivo would lead to decrease in inflammation, glutamate release and improved intracellular glutathione levels. Design/Methods: Monocytes from healthy $(n=8)$, adrenomyeloneuropathy (AMN) $(n=7)$ and ALD patients $(n=5)$ were isolated and derived into macrophages, stimulated with C24:0/C26:0 VLCFAs (equivalent to plasma concentrations in ALD pts) and treated with increasing doses of DNAC, free NAC or dendrimer alone for 6 hours.

Results: Stimulation with VLCFA did not lead to an increase in TNF- \pm or glutamate production or change intracellular glutathione levels in macrophages derived from healthy controls and AMN patients. Macrophages derived from ALD patients had a 3-fold increase in glutamate and TNF- \pm when exposed to VLCFA $(p<0.01)$. ALD macrophages had 2-3 fold lower levels of intracellular glutathione at baseline when compared to AMN and controls, which decreased even further with VLCFA stimulation. D-NAC was more effective than free NAC in bringing glutamate, TNF- \pm and intracellular glutathione to non-stimulated levels.

Conclusion(s): Dendrimer nanotherapies offer a novel method to effectively and selectively target activated macrophages and microglia, providing opportunities for the treatment of neuroinflammatory diseases such as X-ALD.

***

Title: Migraine Prophylaxis using a Novel Caloric Vestibular Stimulation Device

Author: Robert Black, PhD - Scion Neurostim

\section{Abstract:}

A solid-state device has been developed that enables extended CVS therapy in a home setting. This device has been used in a prophylactic randomized controlled trial (RCT) for patients with episodic migraine (NCT01899040). Statistically significant reductions in the number of migraine days in the active group were observed as early as the first treatment month (PP: $-1.9 \pm 0.7$ days, $p=0.01$; ITT: $-1.8 \pm 0.6, p=0.006$ ), and monthly migraine days continued to decrease over the treatment period with a final reduction during the third treatment month of $-3.6 \pm 0.7$ days $(p<0.0001)$ and $-3.3 \pm 0.6$ days $(p<$ 0.0001 ) in the PP and ITT groups, respectively. By contrast, the placebo group did not exhibit a significant reduction in migraine days during any of the treatment months. In a small pilot study, the effects of prolonged caloric vestibular stimulation on cerebral blood flow physiology and autonomic tone were studied. Preliminary results suggest that time-varying CVS thermal waveforms may entrain a pontine-based pacing center that subserves autoregulation. Also, CVS-induced changes in heart rate variability were seen. The viability of various non-invasive metrics in the context of personalized biomarkers for titrating CVS migraine therapy will be discussed. We will present an overview of the CVS neuromodulation approach and review clinical study results to date and ongoing plans for migraine headache trials. CVS efficacy for migraine suggests a mechanism of action that may help to inform theories of migraine pathogenesis. We will also provide an overview of the rich literature background on vestibular pathways and argue that the vestibular system is an ideal candidate for facilitating non-invasive neuromodulation for treating CNS disease.

\section{$* * *$}

Title: Ethoxyquin and Its Novel Analogue Are Effective in Multiple Models of Chemotherapy-induced Peripheral Neuropathies

Authors: Ahmet Hoke MD, PhD; Aysel Fisgun; Nicole Reed - Johns Hopkins School of Medicine; Jing Zhu - Nanjing University of Chinese Medicine
Abstract:
Ethoxyquin (EQ (1,2-dihydro-6-ethoxy-2,2,4,trimethyl qyuinoline)) is an antioxidant used in animal foods for over 50 years. We had identified that EQ is a potent neuroprotective compound against distal axon degenera- tion induced by paclitaxel and shown that this effect is mediated by inhibition of chaperone activity of heat shock protein 90 (Hsp90) (Zhu et.al. Annals of Neurology 2013). More recently we showed that this neuroprotection extends to cisplatin-induced peripheral neuropathy (Zhu et.al. Scientific Reports 2016). In the current study we examined the efficacy and safety of a novel analogue of ethoxyquin called EQ-6. Similar to 
the parent compound, EQ-6 has low toxicity in AMES and genotoxicity tests. Furthermore, in a model of paclitaxel induced peripheral neuropathy, EQ-6 prevented development of behavioral, electrophysiological and morphological outcomes of distal axonal degeneration. This study confirms the role of Hsp90 inhibition as a broad target for neuroprotection and identifies EQ-6 as a potential clinical candidate for prevention of chemotherapy-induced peripheral neuropathy.

$* * *$

Title: Breaking the Code of Fragile X SyndromePathophysiology, Biomarkers, and Therapeutics

Authors: Hao Chen, PhD; Alexie Miagkov; Lisa Leary - DRI Biosciences Corporation

\begin{abstract}
:
Fragile X Syndrome (FXS) is an inherited lifetime intellectual disability and a monogenic cause of autism. Along with various comorbid psychiatric symptoms, the majority of FXS males exhibit mild to severe intellectual disability with an average IQ between $40-50$ and a mental age of $\sim 5$ year-olds. Combining literatures and our recent research, our experimental observations have noted that the pattern of certain protein expressions in the brain of adult murine model of FXS (FMR1KO) resembles the pattern of the same protein expressions of a much younger wild type animal. Accompanying this pattern of protein expressions, another observation was that the blood biochemical profile of an adult FMR1KO resembles that of a much younger wild type animal. Together, these evidences suggest a stalled or delayed cerebral development and maturation. Treatment of the animal models of the FXS with our leading candidate drug ACT01 restored the behavior, rescued cerebral development, and normalized the blood biochemical profiles of the FXS animal models. These evidences suggest that ACT01 is an effective candidate drug useful for treating conditions of FXS; and the blood biochemical profile is a potential prognostic biomarker for the objective assessment of treatment responses.
\end{abstract}

$* * *$

Title: Validation of the Tablet-based Brief Assessment of Cognition (BAC App) for Schizophrenia

Authors: Alexandra S. Atkins, PhD; Brian K. Saxby; Tina Tseng; Adam Vaughan; Philip Harvey, PhD; Meera Narasimhan, PhD; Thomas Patterson, PhD; Richard S.E. Keefe, $\mathrm{PhD}$ - NeuroCog Trials

\section{Abstract:}

Computerized tests benefit from automated scoring procedures and standardized administrator instructions. These methods can reduce the potential for rater error. However, especially in patients with severe mental illnesses and neurologic disorders, the equivalency of traditional and tablet-based tests cannot be assumed. The Brief Assessment of Cognition in Schizophrenia (BACS) is a pen-and-paper cognitive assessment tool that has been used in hundreds of research studies and clinical trials, and has normative data available for generating age- and gender-corrected standardized scores. A tablet-based version of the BACS called the BAC App has been developed. This study compared performance on the BACS and the BAC App in patients with schizophrenia and healthy controls. Test equivalency was assessed, and the applicability of paperbased normative data was evaluated.

Methods: Participants included 48 patients (23 female) with schizophrenia and 50 healthy controls ( 25 female) recruited from three academic sites including the University of California-San Diego, the University of Miami - Miller School of Medicine, and the University of South Carolina. All participants were assessed with the standard pen-andpaper BACS and the BAC App.

Results: In both groups, the distributions of standardized composite scores for the tablet-based BAC App and the pen-and-paper BACS were indistinguishable, and the between-methods mean differences were not statistically significant. The discrimination between patients and controls was similarly robust with the BAC App $(\mathrm{d}=1.34)$ and the BACS $(\mathrm{d}=1.24)$. The between-methods correlations for individual measures in patients were $r>0.70$ except Token Motor $(r=0.43)$ and Tower of London $(r=0.61)$. In patients, performance between the test methods was not significantly different on any test except the Token Motor Test. When data from the Token Motor Test were removed, the between-methods correlation of composite scores improved to $r=.88(\mathrm{df}=48 ; P<.001)$ in healthy controls and $r=.89(\mathrm{df}=46 ; P<.001)$ in patients, consistent with the test-retest reliability of each measure.

Conclusions: The tablet-based BAC App generates results consistent with the traditional pen-and-paper BACS. These data support the notion that the BAC App can now be used in clinical trials and clinical practice.

$* * *$

Title: Assessing Functional Capacity using the UCSD Performance-based Skills Assessment (UPSA-2-VIM) and the Virtual Reality Functional Capacity Assessment Tool (VRFCAT)

Authors: Alexandra S. Atkins, PhD; Vicki G. Davis; Adam Vaughan; Brian K. Saxby; Philip Harvey, PhD; Meera Narasimhan, PhD; Thomas Patterson, PhD; Richard S.E. Keefe, PhD - NeuroCog Trials 


\section{Abstract:}

Reliable measurement of functional capacity is critical in assessing the efficacy of treatments for cognitive impairment in a wide range CNS disorders including schizophrenia, mood disorders, mild cognitive impairment (MCI) and Alzheimer's disease (AD). Despite FDA requirements for functional coprimary endpoints in clinical trials of cognitive enhancing therapies, development and standardization of these functional measures has lagged behind the equally critical cognitive endpoints. In order to optimize signal detection, measures of functional capacity must offer psychometric properties on par with cognitive endpoints. The present research assesses the relative sensitivity and psychometric reliability of two measures of functional capacity, the UCSD Performance-based Skills Assessment (UPSA-2-VIM) and the Virtual Reality Functional Capacity Assessment Tool (VRFCAT).

Methods: Participants included 158 patients with schizophrenia and 166 healthy controls. Participants completed the VRFCAT, UPSA-2-VIM and the MCCB at Visit 1. The VRFCAT and UPSA-2-VIM were completed again at Visit 2. Alternate forms were used for the VRFCAT at Visit 2. Analyses examined the sensitivity, test-retest reliability, and practice effects associated with each measure.

Results: High test-retest reliability was demonstrated for the VRFCAT in both Patients and Controls (ICCs $=0.81$ and 0.65 respectively). Test-retest reliability for the UPSA-2-VIM was also high (ICC $=0.78$ for Patients; $\mathrm{ICC}=0.75$ for Controls). Sensitivity to deficits was similar across the two measures (VRFCAT: $\mathrm{d}=1.21$, $p<.001$; UPSA-2-VIM: $\mathrm{d}=1.16, p<.001$ ), and was consistent with the sensitivity of the MCCB composite $(\mathrm{d}=1.22, p<.001)$. Practice effects were evident for the UPSA-2-VIM $(\mathrm{d}=.35, p<.001)$, but not for the VRFCAT (-.04, ns). The UPSA-2-VIM also demonstrated somewhat increased vulnerability to ceiling effects relative to the VRFCAT, with 8 subjects in the Patient Group performing at ceiling at Visit 1, comparted to 2 on the VRFCAT.

Discussion: Findings demonstrate sensitivity and psychometric reliability of both the UPSA-2-VIM and the VRFCAT. Selection of which measure is best-suited for a given study will likely depend on the study design and population of interest. Although findings suggest increased utility of the VRFCAT as a repeated measure in schizophrenia, the UPSA-2-VIM may be equally well-suited for populations less vulnerable to ceiling and practice effects (e.g. MCI/AD), or for single visit studies.

$* * *$

Title: Preliminary Evidence for the Use of Triple Reuptake Inhibitors (TRIs) for the treatment of PTSD

Authors: Walter Piskorski, MBA; Frank P. Bymaster TRImaran Pharma Inc.; Shane A. Perrine; Aloke K. Dutta Wayne State University, School of Medicine

\section{Abstract:}

Post-traumatic stress disorder (PTSD) is a common disorder which results in marked functional impairment. Two serotonin selective reuptake inhibitors (SSRIs), paroxetine and sertraline, are the only approved drugs for the pharmacotherapy of PTSD, but are only moderately effective and have bothersome adverse events. Neurotransmission of three major neurotransmitters, serotonin (5-HT), norepinephrine (NE), and dopamine (DA), are dysfunctional in PTSD and animal models of traumatic stress exposure, suggesting that multi-modal modulation of these monoamines could result in improved pharmacotherapy of PTSD. Therefore, we have investigated the effects of a triple reuptake inhibitor (TRI) D-578 in rodent models for PTSD. D-578 inhibited NE, 5-HT, and DA transporters with Ki values of 6, 21, and $30 \mathrm{nM}$, respectively, and had low affinity for other off-target neuronal receptors. The effects of D-578 and paroxetine were evaluated in a rat model for traumatic stress exposure - the single prolonged stress (SPS) model - which has been shown to have construct, predictive, and behavioral validity for PTSD. SPS involved exposure of the animals to the serial application of three stressors (restraint, forced swim, ether). Seven days after sham treatment or SPS, rats were treated with vehicle, paroxetine, $(5 \mathrm{mg} / \mathrm{kg})$, or D-578 (10 mg/kg) twice daily. The rats on day 8 were subjected to cued fear conditioning using footshock, followed by extinction (no shock) on day 9 , and extinction retention testing on day 10. SPS had no effect on the acquisition of conditioned fear or extinction behavior compared to sham treatment, but decreased the retention of extinguished fear behavior. D-578, but not paroxetine, attenuated the extinction-retention deficit induced by SPS; but neither drug altered acquisition or extinction learning. These findings suggest that the D-578 has greater efficacy in normalizing extinction-retention learning in a model for PTSD compared to paroxetine. Further support for efficacy of D-578 in pharmacotherapy of PTSD was shown by the finding that D-578, like antidepressants used in PTSD, was highly efficacious in the rat forced swim test for depression, and did not induce hyperlocomotion. Overall these results suggest that D-578 may attenuate retention of fearful memories and be effective in the pharmacotherapy of PTSD.

$* * *$

Title: Network for Excellence in Neuroscience Clinical Trials, NeuroNEXT: Success and Opportunities for Future Collaboration

Authors: Marianne Chase, BA - Massachusetts General Hospital; Dixie Ecklund; Michael Bosch, University of Iowa; Brenda Thornell; Christopher S. Coffey, PhD; Merit Cudkowicz, MD, MSc. - Massachusetts General Hospital

\section{Abstract:}

Background: NeuroNEXT was designed to expand NINDS capability to test promising new therapies, increase efficiency 
of clinical trials, and respond quickly as new opportunities arise to test promising treatments for people with neurological disorders. The Network was funded in Oct 2011 with a goal of conducting 57 Phase 2 trials within 7 years through partnerships with industry, small business and academia. NINDS grant mechanisms attract investigators from industry (X01), academia (U01), and small business (U44). The CCC and DCC work with investigators to develop study design and prepare grant applications which undergo peer review at NINDS. Once funded, CCC and DCC work to operationalize the study: select sites, train investigators, distribute drug and supplies, regulatory compliance, create study database, and implement statistical analyses. Three novel initiatives that increase efficiency in this Network are use of a Central IRB of record, establishment of Master Clinical Trial Agreements, and the availability of experienced clinical trial clinicians and statisticians to help with design activities.

Results: 7As of December 2016, over 150 proposals were submitted to NINDS, 64 were deemed feasible for the Network, 25 initial grants were submitted for peer review, 1 of which was funded without revision. Thirteen grants were revised and resubmitted; 6 have received funding and 1 is pending receipt of funding notification. The first 3 studies; in infants with Spinal Muscular Atropy (SMA), adults with Progressive Multiple Sclerosis (MS) and adults with Myasthenia Gravis (MG); completed enrollment on time. Three studies; in acute Stroke, Huntington's Disease (HD) and Glioblastoma Multiforme (GBM); are currently enrolling and two more; in children with Fragile X Syndrome (FXS) and Cryptogenic Sensory Peripheral Neuropathy (CSPN) are on target to begin enrollment in 2017.

Conclusion: NeuroNEXT has met all of the initial goals within five years of funding; conducting seven studies in partnership with industry (Stroke), academia (SMA, MS, MG, GBM, FXS, CSPN) and small business (HD). The Network sites have demonstrated their capacity to effectively enroll subjects in trials conducted for a variety of neurologic diseases. The Network is primed for future collaborations with industry, academia and small business partners.

***

Title: A Novel Neurometabolic Approach to Treating Alzheimer's Disease. Final Results of a Phase 2a Study of T3D-959 in Mild to Moderate Alzheimer's Subjects

Authors: John Didsbury, PhD; Stanley Chamberlain; Warren Strittmatter; Hoda Gabriel - T3D Therapeutics Inc.

\section{Abstract:}

Background: A key tenet of the metabolic approach to treating Alzheimer's disease (AD) with T3D-959 is that
AD involves a massive positive feedback loop of aberrant glucose and lipid metabolism intertwined with neurodegenerative triggers, e.g. plaques, tangles, inflammation, oxidative stress. T3D-959 is an orally-delivered, PPAR delta/gamma dual nuclear receptor agonist designed to break this feedback loop by improving glucose energy and lipid metabolism dysfunctions in AD.

Methods: 36 subjects with mild-to-moderate AD (MMSE=14-26), were randomized to receive 1 of 4 doses (3mg, 10mg, 30mg, 90mg) of T3D-959 qd for 14-days with 7-day follow-up (FU). Objectives included; evaluation of safety and tolerability, changes in cognitive function measured by Alzheimer's Disease Assessment Scalecognitive subscale (ADAS-cog11) and Digit Symbol Substitution Tests (DSST) and changes in over 800 metabolites in a pharmacometabolomic study.

Results: Results demonstrated that T3D-959 was well tolerated with no safety findings. 32 subjects were evaluable for cognitive testing at end of treatment (EOT), 34 were evaluable at FU. Cognitive improvement, as measured by change in ADAS-cog11 score from baseline were observed in three of four dose groups. A sustained improvement was noted in most doses at FU. Dose trend analysis revealed a significant ApoE4 genotype effect $(p=0.004)$. E4 non-carriers exhibited improved scores at lower doses $(3-30 \mathrm{mg})$ with a range $=-5$ to 6 points change from baseline at FU, while E4 carriers had showed a trend for improvement with increased dose $(30 \mathrm{mg}$, $90 \mathrm{mg}$ ). All subjects, regardless of dose or genotype showed a sustained improvement in DSST scores at FU, average dose group improvement score range $=3.11-6.63$ points . Pharmacometabolomic analysis indicated a dose dependent increase in fatty acid oxidation, and a reduction in markers of insulin resistance. Multiple AD related biomarkers, such as ceramides, plasmalogens, and $\mathrm{N}$-acetylaspartate, showed significant dose dependent changes.

Conclusion: Future clinical studies of T3D-959 are supported by its safety profile, cognitive test results and pharmacometabolomic changes.

Financial Support: This study was supported in part by grant AG-049510 from the NIH.

***

Title: A Neural Bypass Technology to Reconnect the Brain to the Body

Authors: Gaurav Sharma, PhD; Nick Annetta; David Friedenberg - Battelle Memorial Institute; Marcie Bockbrader; W. Mysiw; Ali Rezai - The Ohio State University; Chad Bouton - Feinstein Institute for Medical Research; Herb Bresler - Battelle Memorial Institute 


\section{Abstract:}

Millions of people worldwide suffer from diseases that lead to paralysis through disruption of signal pathways between the brain and the muscles. Neuroprosthetic devices are designed to restore lost function and could be used to form an electronic neural bypass to circumvent disconnected pathways in the nervous system. We have shown that intracortically-recorded signals can be linked in real-time to muscle activation to restore movement in a paralyzed human. The system provided cortical control of isolated finger and hand movements and the participant was also able to use the system to complete functional tasks relevant to daily living.

The investigational system has been demonstrated during an FDA-approved study (Nature, 533:247 250, 2016). We utilized a chronically-implanted microelectrode array to record multiunit activity from the motor cortex in a study participant with $\mathrm{C} 5 / \mathrm{C} 6$ quadriplegia from cervical spinal cord injury (SCI). We applied machine-learning algorithms to decode the neuronal activity and control activation of the participant $\mathrm{s}$ forearm muscles through a custom-built high-resolution neuromuscular electrical stimulation system.

Using the system, the subject achieved multiple voluntary movements of the wrist and hand. The neural signal quality remained suitable for decoding and facilitation of movement over a two-year period. The system provided isolated finger movements and the participant achieved continuous cortical control of six different wrist and hand motions. Clinical assessment showed that when using the system, his motor impairment level improved from $\mathrm{C} 5-\mathrm{C} 6$ to a $\mathrm{C} 7-\mathrm{T} 1$ level unilaterally, conferring on him the critical abilities to grasp, manipulate and release objects. This improvement in function is meaningful for reducing the burden of care in patients with SCI as most $\mathrm{C} 5$ and $\mathrm{C} 6$ patients require assistance for activities of daily living, while C7-T1 level patients can live more independently. The system also enabled volitional control of rhythmic finger movements that are typically coordinated in the spinal cord.

This is the first demonstration of successful control of muscle activation utilizing intracortically-recorded signals in a paralyzed human. In the future, the technology could allow brain-to-body interfacing and bridging of injured portions of the nervous system and have applications in SCI, stroke, traumatic brain injury, and motor neuron disease.

$* * *$

Title: An Overview of Two Late-Stage Studies of Intepirdine, a Novel 5HT6 Receptor Antagonist, for the Treatment of Alzheimer's Disease and Dementia with Lewy Bodies: MINDSET and HEADWAY-DLB

Authors: Shankar Ramaswamy, MD; Lawrence Friedhoff; Ilise Lombardo; Nicholas France; Ebenezer Asare, MD;
Heather Dworak, PhD; Geetha Ramaswamy, MD; Yi Mo, $\mathrm{PhD}$ - Axovant Sciences, Inc. and Roivant Sciences, Inc.

\section{Abstract:}

Introduction: Intepirdine (RVT-101) is a novel 5HT6 receptor antagonist in development for the treatment of Alzheimer's disease (AD) and dementia with Lewy bodies (DLB). The drug candidate has been evaluated in 18 completed clinical trials involving over 2,000 individuals. The largest of these studies was a 48-week randomized placebo-controlled trial of 684 individuals with mild-moderate $\mathrm{AD}$, in which intepirdine demonstrated statistically significant benefits in measurements of cognition (ADAS-cog) and function (ADCS-ADL) versus placebo. This presentation provides an overview of two late-stage clinical trials of intepirdine, including a Phase 3 study in AD and a Phase $2 b$ study in DLB, both of which are ongoing.

Materials and Methods: MINDSET is a Phase 3 study of intepirdine in subjects with mild-moderate AD. In this 24week study, subjects are randomized to receive $35 \mathrm{mg}$ intepirdine or placebo on top of stable background donepezil therapy. Multiple outcome measures are captured during the study, including the ADAS-cog, ADCS-ADL, CIBIC+, Neuropsychiatric Inventory, and Dependence Scale. HEADWAY-DLB is a 24-week Phase $2 b$ study of intepirdine in subjects with DLB. Subjects are randomized to receive $70 \mathrm{mg}$ intepirdine, $35 \mathrm{mg}$ intepirdine, or placebo. Outcome measures include the CIBIC+, a computerized cognitive battery assessment, and a clinical assessment of visual hallucinations. Subjects who complete MINDSET and HEADWAYDLB are eligible to participate in long term extension studies with intepirdine.

Results: MINDSET began in late 2015 and HEADWAY-DLB began in 2016. This presentation will provide further details on both of these studies.

Conclusions: Intepirdine is a 5HT6 receptor antagonist in development for the treatment of AD and DLB. MINDSET is a global Phase 3 clinical trial evaluating intepirdine in $\mathrm{AD}$, and HEADWAY-DLB is a multinational Phase $2 \mathrm{~b}$ study evaluating intepirdine in DLB.

Financial Support: The MINDSET and HEADWAY-DLB studies are sponsored by Axovant Sciences Ltd.

$* * *$

Title: Neuroprotective Methylthiazole Derivatives Rescue Learning and Memory

Authors: Douglas Cowart, PharmD; Doris F. Cully - Revivo Therapeutics, Inc.; Gregory Thatcher - University of Illinois at Chicago 


\section{Abstract:}

Learning and memory deficits in neurodegenerative disease result from synaptic failure and neuronal loss, the latter caused in part by excitotoxicity and oxidative stress. A therapeutic small molecule approach has been developed, directed at restoration of both synaptic function and neuroprotection. Nomethiazole (NMZ) derivatives have been synthesized, based upon a lead neuroprotective pharmacophore acting in part by GABAa receptor potentiation. To provide proof of concept for this new NMZ drug class, selected examples were assayed for: restoration of synaptic function in hippocampal slices from AD transgenic mice; reversal of cognitive deficits, and, brain bioavailability of the prodrug and its neuroprotective methylthiazole metabolite. Prior studies with the prototype NMZ (RIV-5061), indicate that these NO-chimeric Nomethiazoles may be of use in treatment of multiple components of neurodegenerative disorders by acting through pCREB activation and attenuation of TNF-alpha and microglial activation, leading to memory rescue in murine models and attenuation of hallmark AD pathology. Clinical evaluation of the prototype Nomethiazole RIV-5061 is continuing, while preclinical development of the 2 nd generation Nomethiazoles is proceeding.

Financial Support: This work was supported by NIH Grants U01 AGO34294 and 2R42AG044024-02

***

Title: Sativex Associated With Behavioral-Relapse Prevention Strategy as Treatment For Cannabis Dependence: A Pilot Randomized Clinical Trial.

Authors: Jose M. Trigo, PhD - Translational Addiction Research Laboratory, Centre; Alexandra Soliman - Centre for Addiction and Mental Health (CAMH); Lena Quilty Campbell Family Mental Health Research Institute, CAMH; Benedikt Fischer - Social and Epidemiological Research Department, CAMH; Jurgen Rehm; Peter Selby; Allan J. Barnes; Marilyn A. Huestis; Tony George; David Streiner; Gregory Staios; Bernard Le Foll

\begin{abstract}
:
Background: The current lack of pharmacological treatments for cannabis use disorder (CUD) warrants the use of novel approaches and further investigation of promising pharmacotherapy. In this pilot study, we assessed the feasibility of the use of self-titrated dosages of Sativex (1:1," 9-tetrahydrocannabinol (THC)/cannabidiol (CBD) combination) or placebo for the treatment of cannabis dependence among 40 treatment-seeker, community-recruited, cannabisdependent subjects.
\end{abstract}

Methods: Participants underwent a 12 week-treatment double-blinded randomized clinical trial with administration of Sativex (up to $113.4 \mathrm{mg}$ of $\mathrm{THC} /$ $105 \mathrm{mg}$ of CBD) or placebo associated with a weekly behavioural intervention of combined Motivational Enhancement Therapy and Cognitive Behavioral Therapy (MET/CBT). The trial was conducted in compliance with protocol GCP and applicable regulatory requirements. It was approved by the CAMH REB (\#144/2011), Health Canada (control \#152049) and registered on Clinical Trials.gov (ID\# NCT 01747850).

Results: Medication was well-tolerated and no serious adverse events were observed in this study. The number of adverse events did not differ between treatments (F1,34=0.371, NS). There was no significant change in abstinence rates at end of trial. Participants were not able to differentiate between the subjective effects caused by Sativex or placebo treatments $(\mathrm{F} 1,39=0.019$, NS). There was a trend for lower use of cannabis in subjects exposed to high doses Sativex vs. placebo. No significant differences between Sativex and placebo on craving or withdrawal scores were observed.

Conclusions: Sativex in combination with MET/CBT was well tolerated. In addition, our pilot results with high dose Sativex that may reduce cannabis use (with no increases in craving or withdrawal) should be explored in an appropriately powered clinical trial.

Financial Support: Research reported in this publication was supported by the National Institutes of Health (NIH), under award number R21DA031906 (to Dr. Le Foll). The content is solely the responsibility of the authors and does not necessarily represent the official views of the National Institutes of Health.

$* * *$

Title: Improved Thrombolysis via Magnetically Conjugated Delivery cpd

Authors: Francis M. "Duke" Creighton III; Colin Derdeyn, MD - Pulse Therapeutics

\begin{abstract}
:
Background: Of the nearly 800,000 strokes seen in the US every year, most victims do not receive tPA therapy for clot dissolution, primarily due to perceived hemorrhage risks and time delays; of the $\sim 8 \%$ who do, only a $\sim 10 \%$ treatment effect is observed due to tPA therapy $\mathrm{s}$ poor performance. When an artery is occluded by an embolus, flow stagnates. The poor recanalization rates observed with intravenous tPA is likely a function of the time required for diffusion to bring the drug to the clot s surface. The MCD system uses an external magnet and tPA-attached iron particles to improve delivery
\end{abstract}


of tPA to clots. Once the particles arrive at the clot, they continue to generate plasmin as they are mixed with surrounding plasminogen. It is hypothesized that this mechanism will improve lysis in terms of reliability and speed while reducing the tPA dose versus that currently approved. If proven, the MCD technology has the potential to destroy blood clots faster and more reliably while substantially reducing tPA-associated hemorrhage risks, thereby greatly improving neurological outcomes and expanding care beyond what is possible with the current standards of care.

Methods: In vitro experiments were conducted using fibrin clots designed to reproduce thrombolysis of human blood clots. 20ug of MCD particles were used and compared against the physiologically-relevant level of tPA for thrombolysis $(1.2 \mathrm{U} / \mathrm{uL})$, with both placed in direct contact with the clot. Lysis was evaluated at $5 \mathrm{~min}$ intervals out to $4 \mathrm{hrs}$. Results: tPA alone resulted in a lysis rate of $(1.00 \pm 0.05$ (95\% confidence limits)) $\mathrm{mm} / \mathrm{h}(N=13)$. In contrast, MCD resulted in a lysis rate of $(1.90 \pm 0.32) \mathrm{mm} / \mathrm{hr}(N=8)$. Bradford protein assays calculated a maximum per-particle tPA concentration of $(18.33 \pm 0.78) \mathrm{U} / \mathrm{ug}(N=7)$. However, S-2288 activity assays measured an activity of $(2.59 \pm 0.66) \mathrm{U} / \mathrm{ug}(N=6)$. Assuming a 500mg MCD particle dose, a $6 \times$ to $45 \times$ tPA dose reduction appeared to be possible.

Conclusion: The MCD approaches resulted in lysis rates $2 \times$ faster than when tPA was applied directly to the clot. Importantly, efficacy was accomplished using tPAequivalent doses representing at least a 6-fold reduction.

***

Title: Secretory Products of B Cells From Patients With Multiple Sclerosis Mediate Death Of Oligodendrocytes and Neurons Secretory Products of B Cells From Patients With Multiple Sclerosis Mediate Death Of Oligodendrocytes and Neurons Secretory Products of B Cell

Authors: Robert P. Lisak, MD; Liljana Nedelkoska Department of Neurology, Wayne State University School of Medicine; Hanane Touil; Rui Li; Dana Schalk, BSc; Beverly Bealmear, BSc; Gillian Muirhead, PhD; Amit Bar-Or, MD; Joyce A Benjamins, PhD - Department of Neurology, McGill University, Montreal Neurological Institute, Montreal, Quebec, Canada. Department of Neurology, Perelman School of Medicine, University of Pennsylvania, Philadelphia, PA

\footnotetext{
Abstract:

B lymphocytes (B cells) are important in the pathogenesis in all phases of multiple sclerosis (MS). Mechanisms independent of production of immunoglobulins ( $\mathrm{Ig}$ ) or complement activation seem to be involved. Cortical
}

gray matter damage is important in the development of permanent disability and cannot simply be attributed to demyelination and axonal damage in the underlying white matter. Damage occurs in the absence of evidence of Ig or complement activation and is directly proportional to the degree of overlying meningeal inflammation including B cells. We reported that supernatants (Sup) of $B$ cells from the blood of patients with MS induced death of rat oligodendrocytes (OL). B cells from normal controls (NC) did not induce significant cell death. In this study we tested the capacity of B cell Sup of MS patients to induce cell death of both rat $(47.5 \%)$ and human $(55.9 \%)$ neurons in vitro.. There was little cytotoxicity induced by $\mathrm{NC}$ B cell Sup of rat $(9.6 \%)$ or human neurons $(8.8 \%)$. We tested these Sup on rat OL cultures and confirmed our earlier studies, MS B cell Sup were toxic to OL (58\%), NC Sup (4\%) were not. There was no correlation between cytotoxicity and levels of IgG or IgM or with levels of any cytokines, chemokines or associated protein $(n=42)$ as assayed by Luminex and/or ELISA technology. B cell Sup induction of apoptosis of neurons and OL was assessed by visualizing terminal deoxynucleotidyl dUTP nick end labeling (TUNEL) in vitro. Apoptosis of OL was also measured by surface appearance of annexin and expression of activated caspase 3 . There was increase in TUNEL in rat and human neurons induced by MS B cell Sup compared to $\mathrm{NC}$ and increase in TUNEL annexin and activated caspase 3 in OL cultures in response to MS B cell Sup when compared to NC Sup. Current B cell targeted treatments predominately eliminate all B cells from the circulation. Identification of the factor/s secreted by MS B cells might lead to more focused treatment in all phases of MS by inhibiting production of or blocking the toxic effects of these factor/s.

$* * *$

Title: NLY001, A Potential Disease Modifying Agent for Neurodegenerative Diseases

Authors: Seulki Lee, PhD; Russell H. Morgan; Seung Pil Yun; Tae-In Kam; Hanseok Ko; Ted Dawson, MD, PhD Institute for Cell Engineering, Department of Neurology, Johns Hopkins University School of Medicine

\begin{abstract}
:
Parkinson's disease (PD) results primarily from the neurotoxicity caused by the accumulation of Lewy bodies (LB), abnormal deposits of the brain protein alpha-synuclein (asyn). Microglia are a type of glial cells located throughout the brain. During the progression of PD, aggregated a-syn proteins activate microglia to become bad actors. Activated microglial induce reactive astrocytes and release neurotoxic molecules causing neuro-inflammation and neuronal
\end{abstract}


damage. Therefore, a blood-brain-barrier (BBB) penetrant molecule that enables blocking microglial activation and reactive astrocytes formation can potentially halt or prevent PD progression in humans. NLY001 is a differentiated, long-acting glucagon-like peptide-1 receptor (GLP-1R) agonist that penetrates BBB with an extended half-life in nonhuman primates. NLY001 is currently under development as a patient friendly, bi-monthly self-injectable therapy. We have discovered that NLY001 selectively blocks a-syninduced microglial activation and suppresses the formation of reactive astrocytes and production of multiple neurotoxic cytokines that facilitates neuronal cell death, in vitro and in vivo PD models. Unlike other short-acting GLP-1R agonists, NLY001 has the ability to continuously activate GLP-1R in the brain without off-time, which reduces the therapeutic effect during a chronic treatment. Importantly, when two complementary a-syn PD models, a-syn preformed fibrils (PFF)-induced and A53T transgenic (Tg) PD mice, were treated subcutaneously with NLY001, it clearly (1) blocks gliosis, (2) reduces a-syn aggregates/ LB/LN-like pathology, (3) protects dopaminergic neurons, (4) restores behavioral defects and (5) significantly extends the lifespan of Tg PD models. These are surprising results since no GLP-1R agonists demonstrated positive efficacy in genetic PD animal models associated with a-syn pathology. Since we have completed GLP toxicology studies in rodents, we would offer rapid path to investigate NLY001 in clinical proof of concept studies. The overarching goal is to develop a drug with disease-modifying effects and improved patient s compliance for patients with PD and other neurodegenerative diseases including Alzheimer's disease.

***

Title: RNA Trans-splicing: A Gene Reprograming Technology with Major Advantages Over CRISPR for the Development of Neurotherapeutics

Author: Lloyd G. Mitchell, MD - RetroTherapy

\begin{abstract}
:
RetroTherapy s unique RNA repair technology overcomes several of the major obstacles associated with DNA editing and gene therapy, and is especially relevant for the development of neurotherapeutics. RetroTherapy specializes in the design and optimization of proprietary RNA trans-splicing molecules (RTMs) that invade the splicing of a chosen pre-messenger RNA (pre-mRNA) gene target, repairing or completely changing the coding sequence of the resulting mRNA. Gene repair strategies offer 3 main advantages over traditional gene therapy; 1) Size - only a portion of the gene to be repaired is required to be delivered; 2) Expression - the repaired gene retains its natural regulation, thus it is expressed in the right
\end{abstract}

place, amount and time; 3) Dominant negative mutations can be corrected.

RNA trans-splicing provides 2 further advantages over DNA editing/repair methods such as CRISPR-Cas: 1) Size - trans-splicing does not require delivery of any enzyme, such as Cas, which is large and potentially antigenic; 2) Cell division is not required for RNA repair. This is a huge advantage for any potential neurotherapeutic. RTMs have been delivered and expressed by most clinically relevant gene delivery systems, including AAV and lentiviruses. One RTM can replace $>30$ exons, reprograming many 1,000 s of nucleotides. Therefore, 1-3 gene specific RTMs could repair virtually all mutations within any gene and produce full-length repaired protein.

Trans-splicing has corrected tau isoform expression and transport of amyloid precursor protein in human neurons and animal models (frontotemporal dementia and Alzheimer's disease). Other recent studies have shown repair of rhodopsin (retinitis pigmentosa), SMN (spinal motor atrophy), and HTT (Huntington's) in relevant animal models. Trans-splicing has also been used to reprogram genes that cause cancer (p53, CD22, HPV, hCG), cystic fibrosis (CFTR), progeria (lamin A), muscular dystrophy (dystrophin, plectin, titan, dysferlin, DMPK), hemophilia (factor 8), and severe combined immunodeficiency (CD40L, DNA-PK).

RetroTherapy utilizes high-throughput screens that can identify the most efficient and specific RTMs from custom built libraries containing over a million gene specific RTMs. Currently, we are optimizing RTMs as potential therapeutics for leukemia, blindness, Huntington's, Parkinson's, addiction and psychiatric disorders.

Financial Support: RetroTherapy has been funded by grants from NIH, TEDCO, and MD Stem Cell Fund.

$* * *$

Title: Stereopure Nucleic Acid Therapies in Development for the Treatment of Genetic Neurological Diseases

Author: Michael Panzara MD, MPH - WAVE Life Sciences

\begin{abstract}
:
WAVE Life Sciences is a genetic medicines company developing targeted nucleic acid therapies, including therapies for patients impacted by rare diseases. WAVE s proprietary technologies enable the development of oligonucleotide compounds in which both stereochemistry and chemical modification at each position can be precisely controlled. This degree of control enables rational design and synthesis of optimized stereopure oligonucleotides. WAVE s core focus is in neurological diseases, including Huntington's disease (HD) and Duchenne muscular dystrophy (DMD).
\end{abstract}


HD is caused by known mutations on a single gene, characterized by an expansion of a cytosine, adenine, and guanine (CAG) triplet repeat in the Huntingtin (HTT) gene. Each of WVE-120101 and WVE-120102 is an investigational stereopure antisense oligonucleotide (ASO) intended for the treatment of HD. Each of these ASOs selectively target the mutant form of HTT mRNA, specifically acting at the U variant of single nucleotide polymorphisms rs362307 and rs362331, respectively, leaving the wild-type allele (wtHTT) relatively unaffected. In vitro studies have shown that WVE120101 and WVE-120102 selectively induce RNase H cleavage of mHTT over wtHTT, and selectively decrease mHTT mRNA and protein levels compared with wtHTT in multiple cells lines, including patient-derived fibroblasts.

DMD is an X-linked genetic disorder caused predominantly by out-of-frame deletions in the DMD gene, resulting in absent or defective dystrophin protein. Exon-skipping technology has the potential to induce cellular machinery to skip over a targeted exon and restore the reading frame, resulting in the production of internally truncated, but functional, dystrophin protein. WVE-210201 is an investigational stereopure oligonucleotide that specifically induces skipping of exon 51 of DMD pre-mRNA, and is intended for the treatment of DMD. In vitro experiments show that gymnotic delivery (free uptake) of WVE 210201 in DMD patient-derived myotubes induces higher exon 51 skipping efficiency and truncated dystrophin protein restoration compared to stereorandom oligonucleotides. Restoration of the truncated dystrophin protein was quantified to be greater than $50 \%$ of normal skeletal muscle tissue lysates as compared to approximately $1 \%$ with stereorandom oligonucleotides.

WAVE expects to initiate clinical development programs with WVE-120101 and WVE-120102 in HD, and with WVE210201 in DMD, in 2017.

***

Title: FLX-787, a Dual Activator of TRPV1 and TRPA1 Ion Channels, Reduces Muscle Cramp Frequency and Pain Associated with Night Leg Cramps

Authors: Glenn Short, PhD; Jian Liu; Mark Versavel; Brooke Hegarty; Jennifer Szegda; Christoph Westphal, MD, PhD; Thomas Wessel, MD, PhD - Flex Pharma

\footnotetext{
Abstract:

Objectives: Night leg cramps (NLC) are painful supramaximal contractions caused by alpha-motor neuron hyperexcitability and lead to sleep disruptions and reduced quality of life. We have previously demonstrated that activation of TRPV1 and TRPA1 ion channels in the oropharynx and esophagus significantly decrease electrically-induced cramps in healthy adults. We investigated whether this effect would translate to clinical improvements in subjects suffering from NLC. Using a GMP
}

synthesized single molecule that is a naturally-occurring dual TRPV1/TRPA1 activator, FLX-787, we conducted an exploratory cross-over study in subjects with NLC to examine the impact on muscle cramp frequency and pain.

Methods: We investigated two doses of FLX-787 (17 mg and $25 \mathrm{mg}$ ) in an orally-disintegrating tablet (ODT) formulation in a randomized, blinded, placebo-controlled two-part crossover study in otherwise healthy subjects who claimed to experience at least 3 NLCs per week $(n=72)$. At the conclusion of the study, a questionnaire was administered post-hoc to assess the likelihood (not-likely, possible, probable) that participants actually suffered from NLC relative to other conditions such as restless leg syndrome (RLS).

Results: FLX-787 ODT was safe and well-tolerated. Analyses restricted to the first cross-over period of each study part, together with subject selection based on NLC likelihood, demonstrated efficacy. By possible and probable NLC criteria, combined active treatments demonstrated a $\sim 3$-fold decrease in the weekly cramp mean frequency compared to placebo treatments $(p<$ $0.05)$ and an $\sim 8$-fold decrease in cramp pain $(p<0.01)$. As expected for subjects with likely RLS, no difference in cramp frequency or pain was observed.

Conclusion: These results demonstrate that an ODT formulation of FLX-787 may be effective at reducing both cramp frequency and pain associated with NLC, but may not be useful in subjects with RLS. Careful adjudication of selfreported NLC must be performed prior to randomization to avoid confounding data from subjects with other sleep disorders. These hypothesis-generating conclusions, in conjunction with restricting future studies to parallel design to avoid carry-over effects, will allow for improved clinical studies in NLC as well as in neurological indications (ALS, MS, and Charcot-MarieTooth neuropathy) where cramping is prevalent.

$* * *$

Title: Transdermal Fentanyl Abuse and Misuse Deterrence Defent (TM)

Authors: Steven Damon; David Enscore; Alan Smith - 4P Therapeutics

\section{Abstract:}

A transdermal fentanyl patch that offers built-in, passive means to reduce the potential for abuse and misuse would offer a differentiated product over other non-abuse deterrent transdermal patches. The primary routes of abuse of transdermal fentanyl are use of the patch for direct buccal placement or chewing and extraction of the drug from the patch for mucosal (primarily oral) or by injection. Accidental misuse is generally 
via chewing or ingestion of used patches by children or companian animals. Approximately $70 \%$ of all fentanyl patch abuse is by the oral route. Taste aversion addresses primary routes of abuse and accidental misuse. Buccal and/or oral abuse is prevented due to extremely unpleasant bitter taste \& burning sensation and inhaled routes prevented due to highly unpleasant burning sensation to sinus/respiratory tissue.

4P has demonstrated the feasibility of an abuse deterrent patch that offers significant barriers to facile circumvention of the aversive agent-based abuse deterrent patch features. Specifically, the aversive agents are contained in a layer that is bonded strongly to the distal side of the patch backing. Simple techniques to separate the adverse agent containing layer, such as scrapping, are ineffective. The agents and the matrix polymer for the aversive agent layer were selected to yield a biphasic release pattern in aqueous or non-aqueous solvents. The aversive agent release pattern provides rapid release of sufficient quantities of the aversive agents to deter a simple abuse route such as buccal placement of the patch. The rapid aversive agent release is followed by slower release kinetics that prevents rapid elution of the aversive agents after liner removal to separate the fentanyl from the aversive agents.

***

Title: The Benefits of trans-Resveratrol with Polyphenols in Parkinson's Disease

Author: Elizabeth K. Barber, PhD - Barber Innovations LLC

\begin{abstract}
:
Background: Resveratrol, (3,5,4'-trihydroxy-trans-stilbene) is a non-flavonoid polyphenol produced by some plants in response to injury. Most concentrated in red grapes and wine, it coexists with several polyphenols thought to extend its bioavailability. Resveratrol activates the "longevity gene," Sirtuin-1, while the common genetic linkage between Alzheimer's and Parkinson's suggests that resveratrol could reduce Parkinson's symptoms. Red Wine Polyphenols consist of $90 \%$ flavonoids, known to have anti-inflammatory, neuroprotective and anticoagulant properties.
\end{abstract}

Methods: The female patient of 75 years with stage 4 idiopathic Parkinson's, 19 years post-diagnosis, had been followed closely for six years when R/P was first administered. Prior to $\mathrm{R} / \mathrm{P}$, the patient was unable to finish sentences, lacked dyskinesias, had regular hallucinations, an increasing need for $\mathrm{C} / \mathrm{L}$, progressively stooped posture, poor gait and increasing weakness. The patient had plateaued on carbidopa/levodopa 25/100, (C/L 25/100), carbidopa/levodopa 25/100 CR (C/L 25/100 CR) and was given a low dose of an extract of primarily resveratrol with added red wine polyphenols $(\mathrm{R} / \mathrm{P})$ for potential general benefit. After R/P, unexpected dyskinesias and overall functional improvements appeared. Primary outcome measures were discernable as were improvements in cognition and functioning. Pronounced responses necessitated regular measurement of "on/off" state to monitor dyskinesias, tolerance of $\mathrm{R} / \mathrm{P}$ and $\mathrm{C} / \mathrm{L}$ dosages. A regular dosing schedule of R/P was developed and anticoagulation monitored with physician consultation.

Results: The patient exhibited an overnight improvement following the initial dose of $\mathrm{R} / \mathrm{P}$ in cognition and movement requiring a reduced need for carbidopa/levodopa. Progressive improvements in all areas were accompanied by increased dyskinesias requiring lower and more frequent $\mathrm{C} / \mathrm{L}$ doses. Improvements continued for about two months followed by a plateau when R/P was increased. Pronounced dyskinesias necessitated a gradual reduction in $\mathrm{C} / \mathrm{L}$, which over years required a gradual elimination of the $\mathrm{C} / \mathrm{L}$ due to somnolence, improving facial expression and mood. Hallucinations were nearly absent during the five-year period. Relatively small R/P doses indicate that the bioavailability of resveratrol is likely aided by the presence of polyphenols and its own dopaminergic properties. Conclusions: The rapid and progressive patient improvement indicated by UPDRS scores and reduced need for carbidopa/levodopa suggests that $\mathrm{R} / \mathrm{P}$ merits testing in a larger clinical

\section{****}

Title: High-Throughput Screening with Repurposed Medicines Against an SCN8A Mutation Causative for Epilepsy

Authors: Chani M Maher, PhD; Bryant C. Gay; Gregory R. Stewart; JulieAnn Rader; Brett M Antonio; Sonia Santos, MSc; Karen Padilla, MT; Orrin Devinsky, MD; Matt Might, PhD; Steven Petrou, PhD; David B Goldstein, PhD Pairnomix; Aaron C. Gerlach - Icagen Inc.

\begin{abstract}
:
Many patients with an epileptic encephalopathy caused by a genetic mutation are refractory to anti-seizure drugs, highlighting the need for identifying new therapeutic options. Here, we demonstrate an individualized, precision medicine approach combined with high-throughput screening to identify repurposed drug candidates for a patient with a de novo SCN8A R1872Q mutation and a history of early infantile epileptic encephalopathy and global developmental delay. The SCN8A gene encodes the alpha subunit of the voltage-gated Nav1.6 sodium channel, which is essential in the regulation of neuronal excitability. Mutations at the p.1872 site are thought to cause a gain-of-function phenotype in the Nav1.6 channel through increased sodium influx into neurons and subsequent hyper-excitability that is thought to contribute to seizures.
\end{abstract}


Stable clonal cell lines containing either wild-type (WT) SCN8A or SCN8A R1872Q were generated by transfection of DNA into HEK293 cells. An automated patch clamp platform was used to characterize the biophysical properties of WT and mutated Nav 1.6 channels. Cells bearing mutated Nav1.6 sodium channels showed both increased sensitivity to activation and delayed inactivation compared to cells bearing WT Nav1.6 channels, confirming a gain-of-function phenotype for SCN8A R1872Q.

A stimulus-activated, fluorescence-based sodium flux assay, using Asante Natrium Green-2, was employed for high throughput screening studies. A library with $>1,300$ clinically approved drugs was screened, from which 90 lead compounds were identified that significantly inhibited sodium influx in SCN8A R1872Q cells (e2 SD from mean inhibition); a rescreen of the lead compounds recapitulated results from the initial screen.

Subsequent electrophysiological characterization of four lead compounds carbamazepine, carvedilol, amitriptyline and nilvadipine confirmed that the drugs inhibited channel conductance in a concentration-dependent manner in cells containing WT channels or mutated Nav1.6 channels. Although carbamazepine is a known anti-convulsant, the other compounds, amitriptyline (antidepressant), carvedilol (betablocker) and nilvadipine (anti-hypertensive) represent novel drug options for this specific mutation in the Nav1.6 channel. This work underscores the utility of comprehensive screening with precision medicine to identify new therapeutic candidates for rare genetic disorders.

Financial Support: Pairnomix, LLC sponsored this work. $\mathrm{CM}, \mathrm{BG}, \mathrm{JAR}, \mathrm{OD}, \mathrm{MM}, \mathrm{SP}$, and DG have a financial interest in Pairnomix, LLC.

***

Title: Cannabidiol (CBD) Reduces Convulsive Seizure Frequency in Dravet Syndrome: Results of a Multi-center, Randomized, Double-blind, Placebo-controlled Trial (GWPCARE1)

Authors: Orrin Devinsky, MD - NYU Langone Medical Center; J. Helen Cross - UCL Great Ormond Street Institute of Child Health; Linda Laux - Ann and Robert H Lurie Children s Hospital of Chicago; Eric Marsh - The Children s Hospital of Philadelphia; Ian Miller, MD; Rima Nabbout, MD; Ingrid E Scheffer, MD, PhD; Elizabeth A. Thiele, MD; Stephen Wright, MD

\begin{abstract}
:
Objective: Assess the effect of adjunctive CBD for treatment of drug-resistant seizures in Dravet syndrome.
\end{abstract}

Methods: This double-blind, placebo-controlled trial randomized 120 children aged 2-18 years with Dravet syndrome and drug-resistant seizures to receive CBD oral solution $20 \mathrm{mg} / \mathrm{kg} /$ day $(n=61)$ or placebo $(n=59)$ for 14 weeks ( 2 week titration; 12 week maintenance). The primary endpoint was the percentage change from baseline in convulsive seizures (tonic-clonic, tonic, clonic, and atonic) frequency over the 14-week treatment period.

Results: The groups were well-balanced at baseline for demographics. Mean age was 10 years, with $29 \%$ of patients $<6$ years. Patients had previously tried a median 4 AEDs, and were currently taking a median 3 AEDs. Convulsive seizure frequency per month decreased from a median of 12.4 to 5.9 (median reduction of $39 \%$ ) with CBD versus 14.9 to 14.1 (median reduction of $13 \%$ ) with placebo (difference between groups of 23\%; $p=0.012$ ). The proportion of patients with e $50 \%$ reduction in convulsive seizure frequency was $42.6 \%$ with CBD versus $27.1 \%$ with placebo $(\mathrm{OR}=2.0 ; p=0.078)$. Adverse events (AEs) occurred in $93.4 \%$ of $\mathrm{CBD}$ and $74.6 \%$ of placebo patients, and were mostly mild or moderate; the most common were somnolence, diarrhea, and decreased appetite. Serious AEs were reported in $16.4 \%$ of $\mathrm{CBD}$ and $5.1 \%$ of placebo patients, and were considered treatment-related in $8.2 \%$ of CBD patients, all of whom discontinued CBD. Some elevations in transaminases were noted without elevations of bilirubin; all were on concomitant valproate and all resolved. There were no deaths in the study.

Conclusions: In this study, CBD resulted in a significantly greater reduction in convulsive seizure frequency than placebo; adverse events were more frequent with $\mathrm{CBD}$, but it was generally well tolerated.

Financial Support: Funded by GW Research, Ltd; NCT02091375)

$* * *$

Title: Treatment with Cannabidiol (CBD) Significantly Reduces Drop Seizure Frequency in Lennox Gastaut Syndrome (LGS): Results of a Multi-center, Randomized, Double-blind, Placebo-controlled trial (GWPCARE4)

Authors: Elizabeth A. Thiele, MD - Massachusetts General Hospital; Maria Mazurkiewicz-Beldzinska - Medical University of Gdansk; Selim Benbadis - University of South Florida; Eric D. Marsh - Children s Hospital of Philadelphia; Charuta Joshi, MD; Jacqueline A. French, MD; Claire Roberts, PhD, Adam Taylor, PhD; Kenneth Sommerville, MD

\section{Abstract:}

Objective: Evaluate the efficacy of CBD added to antiepileptic drug (AED) therapy for the treatment of seizures associated with LGS. 
Background: Although data have suggested that CBD may be an effective antiepileptic treatment, class 1 evidence supporting its use is lacking. Here we present the first controlled trial of CBD in LGS.

Methods: Eligible patients were 2-55 years old and had a clinical diagnosis of LGS, e8 drop seizures during 4 week baseline (e2/week), and documented failures on e1 AED. Patients were randomized $(1: 1)$ to receive $20 \mathrm{mg} / \mathrm{kg} / \mathrm{day}$ CBD (oral solution) or matched placebo for 14 weeks (2-week titration; 12-week maintenance). The primary efficacy endpoint was percentage change from baseline in drop seizure frequency over the entire 14-week treatment period for patients on CBD vs. placebo.

Results: 171 patients were randomized (86 CBD; 85 placebo); $14 \mathrm{CBD}$ and 1 placebo patient withdrew. Groups were similar at baseline; mean age was 15 years (34\% of patients e 18 years) and median drop seizures/month was 74. Patients had previously taken a median of 6 AEDs, and were taking a median of 3 concomitant AEDs. CBD resulted in a significantly greater median percent reduction in monthly drop seizures than placebo (44\% vs. $22 \% ; p=0.0135$ ) and a significantly greater e $50 \%$ responder rate $(44 \%$ vs $24 \%$; $p=0.0043$ ). The treatment difference was established during the first 4 weeks of the maintenance period. Adverse events (AEs) were reported in $86 \%$ of CBD and $69 \%$ of placebo patients, and were mostly mild or moderate; those $>10 \%$ were diarrhea, somnolence, pyrexia, decreased appetite, and vomiting. Treatment-related serious AEs were reported in $9 \mathrm{CBD}$ patients and 1 placebo patient. Some elevations in transaminases were noted without elevations of bilirubin; most were on concomitant valproate and all resolved. There was 1 death (CBD), considered unrelated to treatment.

Conclusions: Results from this trial suggest that CBD add-on therapy for the treatment of drop seizures associated with LGS may be efficacious, with more AEs than placebo, but generally well tolerated.

Financial Support: Funded by GW Research, Ltd; NCT02224690

$* * *$

Title: High Dose Biotin for Not-active Progressive Multiple Sclerosis

Author: Frederic Sedel MD, PhD - Medday Pharmaceuticals S.A.

\section{Abstract:}

Multiple sclerosis (MS) is a frequent and disabling neurological disease characterized by multifocal destruction of central nervous system myelin. Most patients with MS experience an initial period of relapsing-remitting disease (RRMS) followed 15-20 years later by a progressive disease course (secondary progressive MS; SPMS). Approximately $15 \%$ of patients experience progressive disease from the onset (primary progressive MS; PPMS. Progressive MS, either SPMS or PPMS, can be further categorized as active or not active progressive disease depending on the presence of superimposed inflammatory activity. There are currently no disease-modifying therapies approved for the treatment of not-active progressive MS.

Progressive axonal degeneration in progressive MS is thought to be triggered by a state of virtual hypoxia caused by both chronic demyelination and mitochondrial dysfunction. We recently reported the results of a randomized, double-blind, placebo-controlled study that showed that MD1003 (highdose biotin) achieved sustained reversal of MS-related disability in patients with not active progressive MS $(12.6 \%$ in the active arm vs $0 \%$ in the placebo group, $p=0.005$ ). In addition, a decreased risk of disease s progression (4\% in the active arm vs $13 \%$ in the placebo group, $p=0.07$ ) and better clinical impression of change assessed by the clinical $(p<0.0001)$ and by the patient ( $p=0.0094$ ) were observed after 1 year in the patients treated with MD1003. We hypothesized that MD1003 was exerting a therapeutic effect in progressive MS by reducing neuronal hypoxia. Biotin is a cofactor for acetyl-CoA carboxylase, the rate limiting enzyme that generates malonylCoA, the two-carbon building block for fatty acid synthesis. Biotin is also a cofactor for three enzymes that generate intermediates for the tricarboxylic acid cycle: pyruvate carboxylase, 3-methylcrotonyl-CoA carboxylase and propionyl-CoA carboxylase. Therefore, the improvements in disability seen in patients with progressive MS treated with high-dose biotin may be due to an increase in the supply of precursors for fatty acid synthesis and/or replenishment of the pool of ATP in hypoxic neurons. [1,2]

1. Sedel F, Papeix C, Bellanger A et al. High doses of biotin in chronic progressive multiple sclerosis: A pilot study. Mult Scler Relat Disord 2015;4:159-169.

2. Sedel F, Bernard D, Mock DM, Tourbah A. Targeting demyelination and virtual hypoxia with high-dose biotin as a treatment for progressive multiple sclerosis. Neuropharmacology 2016:110:644-653.

$* * *$

Title: Phase II Study to Assess Safety and Target Engagement of AMX0035, a Novel Combination Therapy, in Alzheimer's Disease

Authors: Kent Leslie, MS; Joshua Cohen; Justin Klee Amylyx Pharmaceuticals; Steven E. Arnold - Massachusetts General Hospital

\section{Abstract:}

Amylyx Pharmaceuticals has developed novel therapeutic, AMX0035, for the treatment of neurodegenerative diseases. 
AMX0035 is a combination of two compounds, Sodium Phenylbutyrate (PB) and Tauroursodeoxycholic Acid (TUDCA). AMX0035 is a proprietary combination of two small molecules, PB and TUDCA, designed to block neuronal death and neurotoxic inflammation through simultaneous inhibition of ER stress and mitochondrial stress. Individually, $\mathrm{PB}$ and TUDCA have been evaluated in in vitro and in vivo models of neurodegenerative disease, as well as clinical trial in ALS, Parkinson's disease, and Huntington's disease. Amylyx discovered a synergy between these two compounds when administered together in a particular range of ratios across multiple preclinical models.

AMX0035 will be entering into a clinical trial in Alzheimer's disease patients in late 2017. The clinical trial will be a sixmonth, parallel-group, randomized, double-blind, placebocontrolled study in volunteers with late mild cognitive impairment (MCI) or early to moderate dementia due to $\mathrm{AD}$. The study is designed to evaluate safety, tolerability, and biomarkers of molecular AD, neurodegeneration and neurophysiology that indicate AMX0035 target engagement and neurobiological effects over 24 weeks. Patients will be evaluated at baseline and at week 24 with multi-sequence structural and functional MRI to assess changes in regional brain volumes (T1), cerebral perfusion (ASL), functional connectivity (BOLD) and cerebrovascular pathology (FLAIR, SWI). Lumbar punctures will be performed at baseline and 24 weeks for selected CSF biomarkers including amyloid- ${ }^{2} 1-42$, tau, and markers of mitochondrial redox, neuronal injury and neuroinflammation. Patients will be evaluated at weeks 1, 12, and 24 for safety, tolerability, and changes in symptoms, as measured with the ADAS-Cog 13, ADCS-ADL and NPI. Success in this trial will provide confidence and insights for a future, larger trial, as well as generate a well-controlled biomarker dataset to inform rational selection of patients and biomarkers in future $\mathrm{AD}$ trials.

$* * *$

Title: Saffron Crocins for Prevention of and Rescue from Retinal Neurodegeneration

Authors: John Paul SanGiovanni, ScD; John M. Davis Davis Laboratories; Silvia Bisti; Rita Maccarone; Stefano De Marco - Università dell Aquila; Marco Piccardi, MD; Benedetto Falsni, MD - Università Cattolica Sacro Cuore, Fondazione Policlinico Gemelli Rome

\footnotetext{
Abstract:

Background: The survival of post-senescent retinal photoreceptors is essential for the process of visual sensation. Metabolic dysfunction, loss of neurotrophic factors, and environmental exposures leading to increased cellular oxidant load and pathologic inflammation are processes implicated in the development and progression of many
}

sight-threatening conditions. In model systems of neurodegenerative disease, bioactive constituents of saffron (crocins and crocetin) act in anti-oxidant and antiapoptotic capacities - these compounds also have been demonstrated to increase oxygen diffusion. Crocin has been shown to alter chromatin structure in a manner that reduces susceptibility to oxidation. Members of our group have demonstrated effects of saffron on: 1) photoreceptor rescue from light damage in vertebrate model systems; and, 2) improvement and subsequent maintenance of central retinal function (flicker sensitivity and visual acuity) in people with early age-related macular degeneration (AMD). Here we extend findings from our AMD study to report preliminary results from work on the efficacy of saffron supplementation in people with ABCA4-related Stargardt s macular dystrophy, an inherited blinding condition characterized by photooxidation-induced loss of cone and rod photoreceptor structure and function.

Methods: IRB-approved, single-center Phase IIa randomized clinical trial (STARSAF02, NCT01278277). Thirtypatient (14 males, 16 females, age range: 15-68 years), 180-day double-masked, placebo-control with cross-over (saffron|placebo) at 90-days. Oral saffron|placebo supplementation at $20 \mathrm{mg} /$ day. Outcomes: Macular cone function measured by focal electroretinogram (fERG) and visual acuity.

Results: fERG stabilization (maintenance of central visual function) occurred after supplementation with saffron, but not placebo. Acuity values within individuals did not differ between interventions at 6 months.

Conclusions: Saffron supplementation shows promise as a potential therapeutic strategy to preserve or rescue retinal photoreceptor cone-mediated function in early AMD and ABCA4-related Stargardt s macular dystrophy.

Financial Support: Telethon Foundation (B. Falsini, M.D.)

$* * *$

Title: Orally-active, Dual-mechanism, Functional Selectivity CNS Ligands as Prospective Alzheimer's Disease-modifying Therapeutics

Authors: Dhwanil A. Dalwadi, PhD - Department of Pharmacology \& Neuroscience, Center; Aurélie Joly-Amado - Department of Molecular Pharmacology \& Physiology, USF Health Byrd Alzheimer's Institute, University of South Florida; Satheesh B. Ravula; Fabio C. Tucci - Epigen Biosciences; Marcia Gordon2, John A. Schetz1,* Department of Pharmacology \& Neuroscience, Center for Neuroscience Discovery, Institute for Healthy Aging, University of North Texas HSC, Fort Worth, TX 76107 


\section{Abstract:}

Unless disease-modifying treatments for Alzheimer's disease (AD) are discovered, the prevalence and the associated financial and caregiver burden of $\mathrm{AD}$ is projected to increase dramatically. To address this urgent unmet medical and societal need, our focus is on discovering drug treatments having the potential to prevent or slow the progression of $\mathrm{AD}$. Brain inflammation triggered by chronic oxidative-nitrosative stress is a proven component in the pathogenic cascade leading to mild cognitive impairment (MCI) and AD. When surplus inflammatory nitric oxide (NO) and superoxide molecules combine they form the brain-impairing reactive species peroxynitrite. This perpetuates inflammation thereby leading to the progressive neurodegeneration seen in AD. Further, cognitive decline is slower in $\mathrm{AD}$ patients with higher levels of CNS brain-derived neurotrophic factor (BDNF), while interference with BDNF trafficking and secretion is associated with an increased risk of AD. Accordingly, our innovative strategy includes simultaneously interrupting the cycle of peroxynitrite generation by blocking unsafe elevations in NO, and enhancing resilience to inflammatory brain insults by facilitating BDNF secretion. The Sigma-1 receptor (S1R) is a stress and ligand-regulated, endoplasmic reticulum chaperone protein which shuttles lipids and proteins to the plasma membrane and is involved in neuroprotective, neurorestorative and neuroplastic responses. Because the $\mathrm{S} 1 \mathrm{R}$ is capable of regulating NO levels and mediating BDNF secretion when brain cells experience high levels of inflammatory stress, we are developing medications that selectively target this protein. By exploiting functionally selective signaling pathways measured with a unique in vitro high throughput platform, we have discovered multiple, orally and CNS-active chemotypes capable of both reducing $\mathrm{NO}$ levels in response to an AD-type stressor and increasing BDNF secretion from neuronal and glial cells. Exploration of the structure-activity space for some of these chemotypes has helped to pinpoint substructural features controlling functionally selective properties. Chronic oral administration of a functionally selective prototype compound significantly improves cognitive performance in a taudepositing transgenic mouse and correlates with reductions in insoluble tau and specific patterns of phosphorylation. These results provide proof-of-concept that S1R ligands with an appropriate functionally selective profile may have therapeutic utility in tau-depositing diseases such as AD and frontotemporal dementia (FTD).

*** $*$

Title: Molecular Vehicle for Intra-Neuronal Delivery of Antibody Fusion Proteins
Author: Philip A Band, PhD - NYU School of Medicine, Hospital for Joint Disease

\section{Abstract:}

We are developing recombinant atoxic derivatives of botulinum neurotoxin (BoNT) as molecular vehicles that can specifically deliver therapeutics to the cytoplasmic compartment of neurons. For proof-of-concept, we have fused an atoxic derivative of full-length BoNT/C1 (BoNT/C1ad) to a single domain antibody (B8) that specifically inactivates the light chain (LC) protease of BoNT/A1. The antibody fusion protein, termed B8-C1ad, was expressed using a baculovirus Sf9 system, and purified to homogeneity using 2-step tandem affinity chromatography. A murine model of BoNT/A intoxication, was developed to test the effectiveness of B8-C1ad as a function of administration time post-exposure to wt BoNT/A. Mice were intoxicated with 2 LD50 of wt BoNT/A by ip administration. At 3, 6, 8, 12, 16 or $20 \mathrm{~h}$ post-intoxication, mice ( $N=10$ per group) were treated ip with either saline, 10 ug B8-C1ad $(0.4 \mathrm{mg} / \mathrm{kg})$, or $1 \mathrm{U}$ of a standard anti-BoNT/A antibody (enough to neutralize 10,000 LD50 of BoNT/A). Treatment with B8-C1ad resulted in $100 \%$ survival when administered up to 12 hours post exposure, compared to the standard antibody which was only $30 \%$ effective by 12 hours. At 16 and 20 hours post-exposure, B8-C1ad treatment resulted in $80 \%$ and $70 \%$ survival, respectively, compared to $0 \%$ survival in the standard antibody groups. The BoNT/Clad molecular vehicle and the B8 antibody alone were ineffective under similar conditions. Surviving animals in the B8-C1ad group resumed weight gain approximately 3-4 days post-treatment, and no rebound of symptoms was observed over a 30 day period. These data demonstrate that B8-C1ad can reverse respiratory symptoms of botulism, and enable survival when administered at times post-exposure when available treatments are ineffective. Importantly, the data strongly suggest that B8-C1ad can inactivate the BoNT/A light chain protease exposed inside the presynaptic compartment of intoxicated neurons. To our knowledge this represents the first example of an antibody specifically delivered to cure an intra-neuronal disease, using a molecular vehicle for delivery instead of a virus. B8-C1ad also addresses the practical need for an anti-botulism therapeutic that can rescue patients exposed to BoNT, when mechanical ventilation is impractical. 